\title{
TOPOLOGICAL CONJUGACIES OF PIECEWISE MONOTONE INTERVAL MAPS
}

\author{
NIKOS A. FOTIADES and MOSES A. BOUDOURIDES
}

(Received 3 January 2000)

\begin{abstract}
Our aim is to establish the topological conjugacy between piecewise monotone expansive interval maps and piecewise linear maps. First, we are concerned with maps satisfying a Markov condition and next with those admitting a certain countable partition. Finally, we compute the topological entropy in the Markov case.
\end{abstract}

2000 Mathematics Subject Classification. Primary 37B10, 37E05, 26 A18.

1. Introduction and preliminaries. Let $I$ be a closed interval in $\mathbb{R}$, which is usually taken to be the interval $[0,1]$, and $f: I \rightarrow I$ a mapping. The iterates of $f$ are the maps $f^{n}$ defined inductively by $f^{0}=\operatorname{id}_{\mathbb{R}}, f^{1}=f, f^{n+1}=f^{n} \circ f$. The (forward or positive) orbit of a point $x \in I$ is the set $O(x)=\left\{f^{n}(x): n \in \mathbb{N}\right\}$. The $\omega$-limit set of $x$ is the set of the limit points of $O(x)$ and is denoted by $\omega(x)$. Two maps $f: I \rightarrow I$ and $g: J \rightarrow J$ ( $J$ a closed interval in $\mathbb{R})$ are called topologically conjugate if there exists a homeomorphism $h: I \rightarrow J$ such that $h \circ f=g \circ h$.

The study of topological conjugacies has commenced with Poincaré in the $1880 \mathrm{~s}$. He considered homeomorphisms $f: S^{1} \rightarrow S^{1}$ of the unit circle $S^{1}=\mathbb{R} / \mathbb{Z}$ with no periodic points and showed that there exist a rotation $R: S^{1} \rightarrow S^{1}$ and a continuous, surjective and monotone map $h: S^{1} \rightarrow S^{1}$ such that $h \circ f=R \circ h$, that is, $f$ and $R$ are topologically semiconjugate. Similar results for piecewise monotone interval maps $f$ were proved later by Parry [10] and Milnor and Thurston [9]. According to them, if $f: I \rightarrow I$ is continuous, piecewise monotone with positive topological entropy $h(f)$, then there exists a piecewise linear map $T:[0,1] \rightarrow[0,1]$ with slope $\pm \exp (h(f))$ such that $f, T$ are topologically semiconjugate. $f$ and $T$ become topologically conjugate, if there are no attracting periodic points and no wandering intervals for $f$. The nonexistence of wandering intervals has been proved for a large class of functions satisfying some mild smoothness conditions (see [3, 6, 7, 8]).

In this paper, we consider the family $\mu$ of functions which are piecewise monotone (but not necessarily continuous) and expansive. Particularly, $f:[0,1] \rightarrow[0,1]$ belongs to the family $\mathcal{M}$ if there exists a partition $0=a_{0}<a_{1}<\cdots<a_{r}=1(r \geq 2)$ of $[0,1]$ such that $f \mid\left[a_{i-1}, a_{i}\right](i=1,2, \ldots, r)$ is a monotone $C^{1}$ function and satisfy the following Markov condition: for every $i=1,2, \ldots, r$, there exist $p(i), q(i) \in\{0,1, \ldots, r\}$ with $p(i)<q(i)$ such that $f\left(a_{i-1}, a_{i}\right)=\left(a_{p(i)}, a_{q(i)}\right)$. Furthermore, we assume that there is $\lambda>1$ such that $\left|f^{\prime}(x)\right| \geq \lambda$, for almost every $x \in[0,1]$, in which case, $f$ is called expansive. Our aim is to show that every $f \in \mathcal{M}$ is topologically conjugate to a map $T$ which is linear on each interval $[(i-1) / r, i / r](i=1,2, \ldots, r)$. Next, we 
consider the class $\mu_{\infty}$ where $[0,1]$ accepts a countable partition accumulating to 1 . Finally, in the last section, we compute the topological entropy for continuous maps in $M$.

NotATion. If $J \subset[0,1]$ is an interval, we denote $|J|$ its length.

2. Topological conjugacies for maps in $\mathcal{M}$. In this section, we study the topological conjugacies for maps $f \in \mathcal{M}$. If $0=a_{0}<a_{1}<\cdots<a_{r}=1$ is the partition corresponding to $f$, we say that $f$ is of order $r$. The points of the partition are called critical points of $f$. We denote by $I_{1}, \ldots, I_{r}$ the intervals of the partition, that is, $I_{j}=\left(a_{j-1}, a_{j}\right)$. We assume that these intervals are maximal in the sense that if $I$ is an interval which strictly contains one of them, then $f \mid I$ is neither continuous nor monotone. Also, we denote by $f_{j}$ the restriction of $f$ to $I_{j}$. Finally, we denote by $F_{j_{1} j_{2} \cdots j_{k}}$ the composition $f_{j_{1}}^{-1} \circ f_{j_{2}}^{-1} \circ \cdots \circ f_{j_{k}}^{-1}$. Note that $F_{j_{1} j_{2} \cdots j_{k}}$ is not necessarily defined for every (finite) sequence $j_{1} j_{2} \cdots j_{k}$. Moreover, $F_{j_{1} j_{2} \cdots j_{k}}(x)$ is the unique point $y \in I_{j_{1}}$ such that $f(y) \in I_{j_{2}}, \ldots, f^{k-1}(y) \in I_{j_{k}}$ and $f^{k}(y)=x$.

An open interval $J \subset[0,1]$ is called a branch of $f^{n}$ if $f^{n} \mid J$ is continuous, monotone and $J$ is maximal with these properties. The set of branches of $f^{n}$ is denoted by $B_{n}(f)$. Moreover, we define the sets

$$
\begin{gathered}
\mathscr{C}_{n}(f)=\bigcup_{j=0}^{r} \bigcup_{i=0}^{n-1} f^{-i}\left(a_{j}\right), \quad n=1,2, \ldots, \\
\mathscr{C}(f)=\bigcup_{j=0}^{r} \bigcup_{i=0}^{\infty} f^{-i}\left(a_{j}\right) .
\end{gathered}
$$

Frequently, we write $\mathscr{C}_{n}$ and $\mathscr{C}$ instead of $\mathscr{C}_{n}(f)$ and $\mathscr{C}(f)$.

In what follows, we introduce some notions from symbolic dynamics. To each point $x$ of $\mathscr{C}$, there corresponds a sequence of symbols which is related with the order of the points of $O(x)$.

DEFINITION 2.1. The itinerary of $x \in \mathscr{C}$ with respect to $f \in \mathcal{M}$ is a sequence $\underline{i}_{f}(x)=$ $\left\{i_{n}(x)\right\}_{n=0}^{\infty}$, where

$$
i_{n}(x)= \begin{cases}j, & \text { if } f^{n}(x) \in I_{j}, \\ \frac{2 j+1}{2}, & \text { if } f^{n}(x)=a_{j} .\end{cases}
$$

An interesting notion in symbolic dynamics is the shift map $\sigma$ : if $\underline{x}=\left\{x_{n}\right\}_{n=0}^{\infty}$, then $\sigma(\underline{x})=\underline{y}$, where $\underline{y}=\left\{x_{n}\right\}_{n=1}^{\infty}$. Inductively, we have $\sigma^{k}(\underline{x})=\left\{x_{n}\right\}_{n=k}^{\infty}$. To each $f \in \mathcal{M}$ of order $r$, we associate a subset of $\{1 / 2,1,3 / 2, \ldots, r,(2 r+1) / 2\}^{\mathbb{N}}$. We describe this set in the following definition.

DEFINITION 2.2. Let $f \in \mathcal{M}$ with partition $0=a_{0}<a_{1}<\cdots<a_{r}=1$. We define the set of sequences $\Sigma(f)=\left\{\underline{a}: \underline{a}=\left\{x_{n}\right\}_{n=0}^{\infty}\right\}$ with entries from the set $\{1 / 2,1,3 / 2, \ldots, r$, $(2 r+1) / 2\}$, which satisfy the following conditions:

(i) Let $\underline{a}=\left\{x_{n}\right\} \in \Sigma(f)$. Then there exists an entry $x_{n}$ of $\underline{a}$ of the form $(2 k+1) / 2$, where $k=0,1, \ldots, r$. Furthermore, if $x_{N}$ is the first entry of $\underline{a}$ with this property, then $\sigma^{N}(\underline{a})=\underline{i}_{f}\left(a_{k}\right)$. 
(ii) If $n<N-1$ and $x_{n}=j$, then $p(j)+1 \leq x_{n+1} \leq q(j)$.

It is possible to define an order on the set $\underline{i}_{f}(\mathscr{C})$ which is consistent with the natural order of real numbers. Two sequences of symbols $\underline{x}=\left\{x_{n}\right\}_{n=0}^{\infty}$ and $\underline{y}=\left\{y_{n}\right\}_{n=0}^{\infty}$ belonging to $\{1 / 2,1,3 / 2, \ldots, r,(2 r+1) / 2\}^{\mathbb{N}}$ are called to have discrepancy $n$ if $x_{i}=y_{i}$, for $i=0,1, \ldots, n-1$, and $x_{n} \neq y_{n}$. If the itineraries of two points of $\mathscr{C}$ have discrepancy $n$, then the first $n$ points of their orbits are visiting simultaneously the same intervals of $B_{1}(f)$. Moreover, we define $1 / 2 \prec 1 \prec 3 / 2 \prec \cdots \prec r \prec(2 r+1) / 2$.

DEFINITION 2.3. Let $f \in \mathcal{M}$ and $x, y \in \mathscr{C}$ with $x \neq y$. We assume that itineraries $\underline{i}_{f}(x)$ and $\underline{i}_{f}(y)$ have discrepancy $n$ and that $f$ is decreasing in $k$ common intervals.

(i) When $k$ is even, then $\underline{i}_{f}(x) \prec \underline{i}_{f}(y)$ if and only if $i_{n}(x) \prec i_{n}(y)$.

(ii) When $k$ is odd, then $\underline{i}_{f}(x) \prec \underline{i}_{f}(y)$ if and only if $i_{n}(y) \prec i_{n}(x)$.

LEMmA 2.4. Let $f \in \mathcal{M}$ be of order $r$ and let $x, y \in \mathscr{C}$ with $x \neq y$. Then $\underline{i}_{f}(x) \prec \underline{i}_{f}(y)$ if and only if $x<y$.

Proof. We assume that itineraries $\underline{i}_{f}(x)$ and $\underline{i}_{f}(y)$ have discrepancy $n$. That is, $i_{k}(x)=i_{k}(y)=j_{k}$, for $k=0,1, \ldots, n-1$, and $i_{n}(x) \neq i_{n}(y)$. We claim that $j_{0}, j_{1}, \ldots$, $j_{n-1}$ are not of the form $(2 s+1) / 2$. To prove this, we assume the contrary, whence $\underline{i}_{f}(x)=\underline{i}_{f}(y)$, which is a contradiction, since $i_{n}(x) \neq i_{n}(y)$. From Definition 2.1, $x, y$ belong to $I_{j_{0}}$ and successively visit the intervals $I_{j_{1}}, \ldots, I_{j_{n-1}}$. So, we can write $x=F_{j_{0} j_{1} \cdots j_{n-1}}\left(f^{n}(x)\right)$ and $y=F_{j_{0} j_{1} \cdots j_{n-1}}\left(f^{n}(y)\right)$. We assume that $f$ is decreasing in $k$ intervals among $I_{j_{0}}, I_{j_{1}}, \ldots, I_{j_{n-1}}$. There are two cases.

(i) When $k$ is even, then $F_{j_{0} j_{1} \cdots j_{n-1}}$ is increasing. Assume that $\underline{i}_{f}(x) \prec \underline{i}_{f}(y)$, then from Definition 2.3 we have $i_{n}(x) \prec i_{n}(y)$. This means that $f^{n}(x)<f^{n}(y)$ and, hence, $x=F_{j_{0} j_{1} \cdots j_{n-1}}\left(f^{n}(x)\right)<y=F_{j_{0} j_{1} \cdots j_{n-1}}\left(f^{n}(y)\right)$.

(ii) When $k$ is odd, then $F_{j_{0} j_{1} \cdots j_{n-1}}$ is decreasing. Assume that $\underline{i}_{f}(x) \prec \underline{i}_{f}(y)$, then from Definition 2.3 we have $i_{n}(y) \prec i_{n}(x)$. This means that $f^{n}(x)>f^{n}(y)$ and, hence, $x=F_{j_{0} j_{1} \cdots j_{n-1}}\left(f^{n}(x)\right)<y=F_{j_{0} j_{1} \cdots j_{n-1}}\left(f^{n}(y)\right)$.

LEMMA 2.5. Let $f \in \mathcal{M}$ be of order $r$. The map $\underline{\underline{i}}_{f}: \mathscr{C} \rightarrow \Sigma(f)$ is a bijection.

Proof. Let $x, y \in \mathscr{C}$ with $\underline{i}_{f}(x)=\underline{i}_{f}(y)$. Let $k, m$ be the minimal integers for which $f^{k}(x), f^{m}(y)$ are critical points of $f$. Assume that $k \neq m$ (let $k<m$ ). Since $f^{k}(x)$ is a critical point, then $f^{k+1}(x)=0$ or 1 , and, so, $i_{k+1}(x)=1 / 2$ or $(2 r+1) / 2$. On the other hand, $i_{k}(y)=1,2, \ldots, r$, and, hence, $i_{k+1}(y) \neq 1 / 2$ and $i_{k+1}(y) \neq(2 r+1) / 2$, which is a contradiction, since $i_{k+1}(x)=i_{k+1}(y)$. So, $k=m$. Furthermore, we observe that $f^{k}(x)=f^{k}(y)$, since $i_{k}(x)=i_{k}(y)$ and it is of the form $(2 j+1) / 2$. Consequently, $f^{k}(x)=f^{k}(y)=a_{j}$.

Assume that $i_{n}(x)=i_{n}(y)=j_{n} \in \mathbb{N}$, for $n=0,1, \ldots, k-1$. From Definition 2.1, $x, y$ belong to $I_{j_{0}}$ and successively visit the intervals $I_{j_{1}}, \ldots, I_{j_{k-1}}$. So, we can write $x=F_{j_{0} j_{1} \cdots j_{k-1}}\left(f^{k}(x)\right)$ and $y=F_{j_{0} j_{1} \cdots j_{k-1}}\left(f^{k}(y)\right)$. Since $f^{k}(x)=f^{k}(y)$, we have $x=y$. Thus, $\underline{i}_{f}$ is injective.

Let $\underline{a}=\left\{x_{n}\right\} \in \Sigma(f)$. We shall show that there exists an $x \in \mathscr{C}$ such that $\underline{i}_{f}(x)=\underline{a}$. From Definition 2.2, an entry of the sequence $\underline{a}$ is of the form $(2 k+1) / 2$. Let $x_{n}$ be the first entry with this property. Then $x=F_{x_{0} x_{1} \cdots x_{n-1}}\left(a_{k}\right)$ satisfies the desired property. 
Proposition 2.6. Let $f \in \mathcal{M}$ be of order $r$. Then $\mathscr{b}$ is dence in $[0,1]$.

Proof. Let $\tilde{J} \subset[0,1]$ be an open interval such that $\tilde{J} \cap \mathscr{C}=\varnothing$. First, we show that $f^{n}(\tilde{J}) \cap \mathscr{C}=\varnothing$, for $n \in \mathbb{N}$. We assume, in the contrary, that there exists $x \in f^{n}(\tilde{J}) \cap \mathscr{C}$, then there is $y \in \tilde{J}$ such that $x=f^{n}(y)$. But, $f^{m}(x)=a_{k}$, for some $m \in \mathbb{N}$ and $k=0,1,2, \ldots, r$, since $x \in \mathscr{C}$. So, $f^{m+n}(y)=f^{m}(x)=a_{k}$, that is, $y \in \mathscr{C}$, which is a contradiction, since $\tilde{J} \cap \mathscr{C}=\varnothing$.

As $f^{n}(\tilde{J}) \cap \mathscr{C}=\varnothing$, for $n \in \mathbb{N}$, it turns out that $f$ is monotone and $C^{1}$ on each interval $\tilde{J}, f(\tilde{J}), f^{2}(\tilde{J}), \ldots$

We prove by induction that $\left|f^{n}(\tilde{J})\right| \geq \lambda^{n}|\tilde{J}|$, for $n \geq 1$. From the mean value theorem and since $f \mid \tilde{J}$ is monotone, we have $|f(\tilde{J})| /|\tilde{J}|=\left|f^{\prime}(a)\right|$, for some $a \in \tilde{J}$. But, $\left|f^{\prime}(a)\right| \geq \lambda$ and, hence $|f(\tilde{J})| \geq \lambda|\tilde{J}|$. We assume that the claim is true for $k<n$. From the mean value theorem and since $f \mid f^{n-1}(\tilde{J})$ is monotone, we have $\left|f^{n}(\tilde{J})\right| /\left|f^{n-1}(\tilde{J})\right|$ $=\left|f^{\prime}\left(a_{1}\right)\right| \geq \lambda$, for some $a_{1} \in f^{n-1}(\tilde{J})$. From the induction assumption, we have $\left|f^{n-1}(\tilde{J})\right| \geq \lambda^{n-1}|\tilde{J}|$. Combining the last two inequalities, we have $\left|f^{n}(\tilde{J})\right| \geq \lambda^{n}|\tilde{J}|$.

Thus, for some $n \in \mathbb{N}, \lambda^{n}|\tilde{J}|>1$, which is a contradiction, since $\left|f^{n}(\tilde{J})\right| \leq 1$.

THEOREM 2.7. Let $f \in \mathcal{M}$ be of order $r$ with partition $0=a_{0}<a_{1}<\cdots<a_{r}=1$. We consider the map $T \in \mathcal{M}$ with partition $0<1 / r<2 / r<\cdots<(r-1) / r<1$ which is linear in each interval $[(i-1) / r, i / r]$ and $T((i-1) / r, i / r)=(p(i) / r, q(i) / r)$. Furthermore, $T \mid[(i-1) / r, i / r]$ is of the same monotonicity type with $f \mid\left[a_{i-1}, a_{i}\right]$ and it is continuous, from the right or from the left at $i / r$, when $f$ is continuous, from the right or from the left at $a_{i}$, respectively. Then $f$ and $T$ are topologically conjugate. (Figure 2.1)

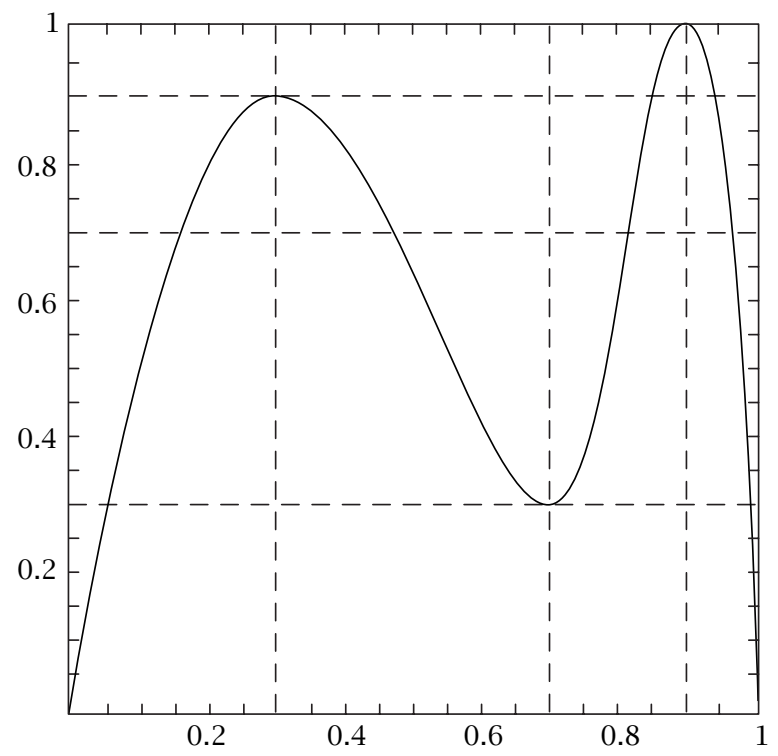

FIGURE 2.1.

Proof. From Definition 2.2, we have $\Sigma(f)=\Sigma(T)$. With this observation and since $\underline{i}_{f}$ and $\underline{i}_{T}$ are bijections (Lemma 2.5), we can define a correspondence $h: \mathscr{b}(f) \rightarrow$ 
$\mathscr{C}(T)$, which is an order preserving bijection and such that $h \circ f=T \circ h$. For $x \in$ $\mathscr{C}(f)$, we define $h(x)$ to be the unique element of $\mathscr{C}(T)$, for which $\underline{i}_{f}(x)=\underline{i}_{T}(h(x))$. Equivalently, $h=\underline{i}_{T}^{-1} \circ \underline{i}_{f}$. But since $\underline{i}_{f}$ and $\underline{i}_{T}$ are bijections, we have that $h$ is also a bijection. From Lemma 2.4, $\underline{i}_{f}$ and $\underline{\underline{i}}_{T}$ are order preserving maps and, so, the same holds for $h$.

Let $x \in \mathscr{b}(f)$. We shall show that $h \circ f(x)$ and $T \circ h(x)$ have the same itinerary with respect to $T$. Indeed,

$$
\underline{i}_{T}(h(f(x)))=\underline{i}_{f}(f(x))=\sigma\left(\underline{i}_{f}(x)\right) .
$$

On the other hand,

$$
\underline{i}_{T}(T(h(x)))=\sigma\left(\underline{i}_{T}(h(x))\right)=\sigma\left(\underline{i}_{f}(x)\right) .
$$

Since $\underline{\boldsymbol{i}}_{T}$ is an injection, we have that $h \circ f(x)=T \circ h(x)$.

Since $\mathscr{C}(f)$ and $\mathscr{C}(T)$ are dense in [0,1] (Proposition 2.6), $h$ can extend to a homeomorphism $\tilde{h}:[0,1] \rightarrow[0,1]$ such that $\tilde{h} \circ f=T \circ \tilde{h}$.

3. Topological conjugacies for maps in $\mu_{\infty}$. In the previous sections, we had studied functions with a finite partition. Here we study a special class of functions with countable partition. Some modifications are necessary.

DEFINITION 3.1. A map $f:[0,1] \rightarrow[0,1]$ belongs to the class of functions $\mu_{\infty}$ if there exists a sequence of real numbers $\left\{a_{n}\right\}_{n=0}^{\infty}$ with $0=a_{0}<a_{1}<a_{2}<\cdots$ and $\lim _{n \rightarrow \infty} a_{n}=1$ such that:

(i) $f$ is $C^{1}$ and monotone on each interval $\left[a_{i-1}, a_{i}\right]$ of the partition.

(ii) For every $i \in \mathbb{N}^{*}$, there exist unique $p(i), q(i) \in \mathbb{N}$ such that $f\left(a_{i-1}, a_{i}\right)=$ $\left(a_{p(i)}, a_{q(i)}\right)$.

(iii) There exists $\lambda>1$ such that $\left|f^{\prime}(x)\right| \geq \lambda$, for every $x \neq a_{i}$.

In this case, $\mathscr{C}(f)=\cup_{j=0}^{\infty} \cup_{i=0}^{\infty} f^{-i}\left(a_{j}\right)$.

DEFINITION 3.2. Let $f \in \mathcal{M}_{\infty}$ with partition $0=a_{0}<a_{1}<a_{2}<\cdots<1$. We define the set of sequences $\Sigma_{\infty}(f)=\left\{\underline{a}: \underline{a}=\left\{x_{n}\right\}_{n=0}^{\infty}\right\}$ with entries from $\{1 / 2,1,3 / 2, \ldots\}$, which satisfy the following conditions:

(i) Let $\underline{a}=\left\{x_{n}\right\} \in \Sigma_{\infty}(f)$. Then there exists an entry $x_{n}$ of $\underline{a}$, of the form $(2 k+1) / 2$, where $k=0,1, \ldots$. Furthermore, if $x_{N}$ is the first entry of $\underline{a}$ with this property, then $\sigma^{N}(\underline{a})=\underline{i}_{f}\left(a_{k}\right)$.

(ii) If $n<N-1$ and $x_{n}=j$, then $p(j)+1 \leq x_{n+1} \leq q(j)$.

THEOREM 3.3. Let $f \in M_{\infty}$ with partition $0=a_{0}<a_{1}<a_{2}<\cdots<1$. We consider the map $T \in M_{\infty}$ with partition $0<1 / 2<2 / 3<3 / 4<\cdots<1$ which is linear in each interval $[(i-1) / i, i /(i+1)]$ and $T((i-1) / i, i /(i+1))=(p(i) /(p(i)+1), q(i) /(q(i)+1))$. Furthermore, $T \mid[(i-1) / i, i /(i+1)]$ is of the same monotonicity type with $f \mid\left[a_{i-1}, a_{i}\right]$ and it is continuous, from the right or from the left at $i /(i+1)$, when $f$ is continuous, from the right or from the left at $a_{i}$, respectively. Then $f$ and $T$ are topologically conjugate.

Proof. The proof of this theorem is the same as the proof of Theorem 2.7. 
4. Computation of topological entropy for continuous Markov maps. Topological entropy is a measure of the dynamical complexity of a map and it is a topological invariant. There is an important theorem connecting topological entropy with the number $c_{n}$ of maximal intervals of monotonicity of the iterate $f^{n}$ (see $[1,4]$ ).

THEOREM 4.1 (Misiurewicz-Szlenk). Let $f: I \rightarrow I$ be a continuous, piecewise monotone map. Then the topological entropy of $f$ is equal to the number

$$
\lim _{n \rightarrow \infty} \frac{1}{n} \ln c_{n}
$$

As a corollary of the above theorem, if $f$ is a piecewise linear map with slope $\pm s$, then the topological entropy of $f$ is equal to $\max \{0, \ln s\}$.

Let $f$ be a continuous map in $\mathcal{M}$ and $T$ as in Theorem 2.7. The slope of $T$ is not necessarily constant. Observe that Theorem 2.7 still holds if we change the partition $0<1 / r<2 / r<\cdots<(r-1) / r<1$ with any other partition $0=b_{0}<b_{1}<\cdots<b_{r}=1$ of $[0,1]$. So, it is natural to ask the following question. Can we find a partition $0=$ $b_{0}<b_{1}<\cdots<b_{r}=1$ of $[0,1]$ such that $\left|b_{q(i)}-b_{p(i)}\right| /\left(b_{i}-b_{i-1}\right)$ is constant?

To answer this question, to each $f \in \mathcal{M}$, we associate an $r \times r$ matrix $A=\left[a_{i j}\right]$ defined by

$$
a_{i j}= \begin{cases}0, & \text { if }\left(b_{i-1}, b_{i}\right) \cap f^{-1}\left(b_{j-1}, b_{j}\right)=\varnothing, \\ 1, & \text { if }\left(b_{i-1}, b_{i}\right) \cap f^{-1}\left(b_{j-1}, b_{j}\right) \neq \varnothing .\end{cases}
$$

Observe that $A$ is nonnegative. According to the Perron-Frobenius theorem, there exists a unique nonnegative eigenvalue $s \geq 0$, which is maximal in absolute value among all the other eigenvalues and corresponding to a nonnegative eigenvector (see Gantmacher [5]).

Proposition 4.2. Assume that $f \in \mathcal{M}$ is a continuous map of order $r, A$ is the corresponding matrix, and $s$ is the "maximal" eigenvalue of $A$.

(a) If $s>1$ and the corresponding eigenvector is positive, then the topological entropy of $f$ is $\ln s$.

(b) If $s \leq 1$ or at least one component of the corresponding eigenvector is zero, then the topological entropy of $f$ is zero.

Proof. (a) Assume that there exist a partition $0=b_{0}<b_{1}<\cdots<b_{r}=1$ and a constant $s>1$ such that $\left|T\left(b_{i-1}, b_{i}\right)\right|=s\left|\left(b_{i-1}, b_{i}\right)\right|$, for $i=1,2, \ldots, r$. If we let $x_{i}=$ $b_{i}-b_{i-1}>0$, the above relation gives

$$
x_{p(i)+1}+x_{p(i)+2}+\cdots+x_{q(i)}=s x_{i}, \quad i=1,2, \ldots, r,
$$

or, equivalently,

$$
A x=s x \text {, where } x=\left(x_{1}, \ldots, x_{r}\right)^{\top} .
$$

Thus, there exist a partition $0=b_{0}<b_{1}<\cdots<b_{r}=1$ and a constant $s>1$ such that $\left|T\left(b_{i-1}, b_{i}\right)\right|=s\left|\left(b_{i-1}, b_{i}\right)\right|$, for $i=1,2, \ldots, r$, if and only if (a) holds.

(b) Assume on the contrary that $h(f)>0$. Then $f$ is conjugate to a piecewise linear 
map with constant slope [9]. It follows that there exist a partition $0=b_{0}<b_{1}<\cdots<$ $b_{r}=1$ and a constant $s>1$ such that $\left|T\left(b_{i-1}, b_{i}\right)\right|=s\left|\left(b_{i-1}, b_{i}\right)\right|$, for $i=1,2, \ldots, r$. This is equivalent to (a), which contradicts (b).

REMARK 4.3. There is a similar result in [2]. The proof we give here is more simple and is based heavily on Theorem 2.7.

The above proposition gives a method to construct the partition $0=b_{0}<b_{1}<$ $\cdots<b_{r}=1$, when we are in case (a). Assume that $\left(u_{1}, u_{2}, \ldots, u_{r}\right)^{\top}$ is an eigenvector corresponding to the maximal eigenvalue. Then $b_{0}=0$ and

$$
b_{k}=\frac{\sum_{i=1}^{k} u_{i}}{\sum_{i=1}^{r} u_{i}} \text { for } k=1,2, \ldots, r .
$$

Consider the map $f \in \mathcal{M}$ whose graph is shown in Figure 2.1. According to Theorem 2.7, $f$ is topologically conjugate with $T$ which is piecewise linear (the graph of $T$ is shown in Figure 4.1). The associated matrix to $f$ is

$$
A=\left[\begin{array}{llll}
1 & 1 & 1 & 0 \\
0 & 1 & 1 & 0 \\
0 & 1 & 1 & 1 \\
1 & 1 & 1 & 1
\end{array}\right] .
$$

The maximal eigenvalue is $s=2.8393$ and an eigenvector is

$$
(0.6478,0.4196,0.7718,1)^{\top} .
$$

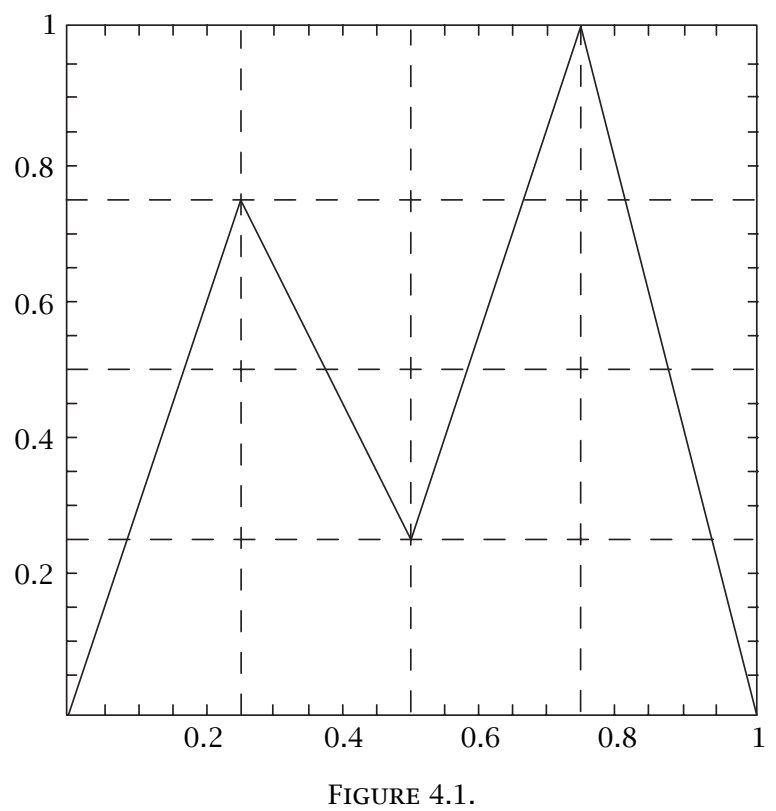




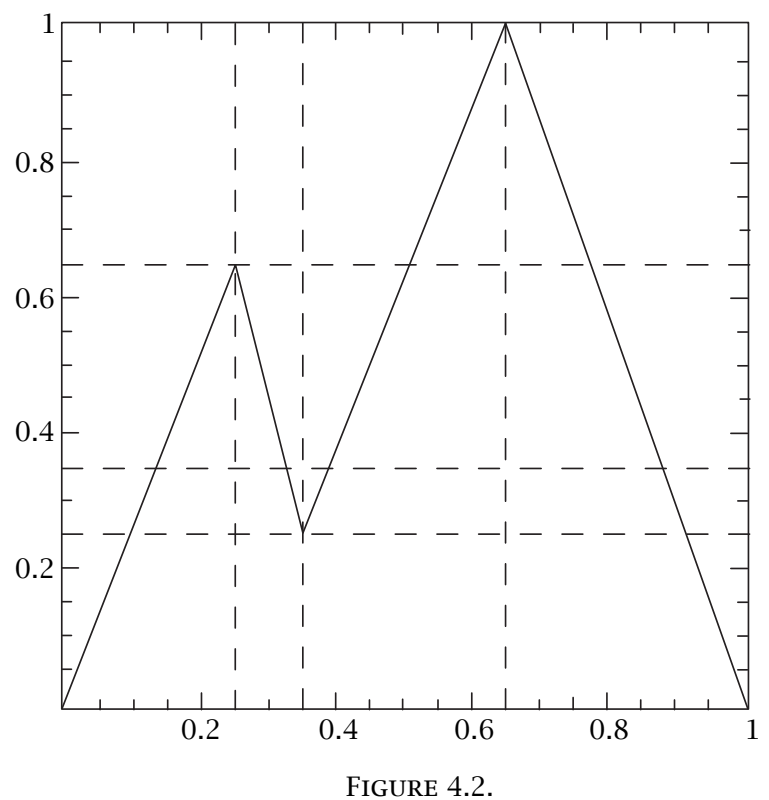

Then from (4.5) we have $b_{0}=0, b_{1}=0.2282, b_{2}=0.3759, b_{3}=0.6478, b_{4}=1 . f$ is topologically conjugate to $T^{\prime}$ whose graph is shown in Figure 4.2. Since the slope of $T^{\prime}$ is constant in absolute value we have that $h(f)=\ln s=1.0435$.

ACKNOWLEDGEMENT. This research was supported by the State Scholarship Foundation of Greece.

\section{REFERENCES}

[1] L. Alsedà, J. Llibre, and M. Misiurewicz, Combinatorial Dynamics and Entropy in Dimension One, Advanced Series in Nonlinear Dynamics, vol. 5, World Scientific Publishing Co., Inc., River Edge, NJ, 1993. MR 95j:58042. Zbl 843.58034.

[2] L. S. Block and W. A. Coppel, Dynamics in One Dimension, Lecture Notes in Math., vol. 1513, Springer-Verlag, Berlin, 1992. MR 93g:58091. Zbl 746.58007.

[3] A. M. Blokh and M. Y. Lyubich, Nonexistence of wandering intervals and structure of topological attractors of one-dimensional dynamical systems. II. The smooth case, Ergodic Theory Dynamical Systems 9 (1989), no. 4, 751-758. MR 91e:58101. Zbl 665.58024.

[4] W. de Melo and S. van Strien, One-dimensional Dynamics, Ergebnisse der Mathematik und ihrer Grenzgebiete (3) [Results in Mathematics and Related Areas (3)], vol. 25, Springer-Verlag, Berlin, 1993. MR 95a:58035. Zbl 791.58003.

[5] F. R. Gantmacher, The Theory of Matrices. Vols. 1, 2, Translated by K. A. Hirsch, Chelsea Publishing Co., New York, 1959. MR 21\#6372c. Zbl 927.15002.

[6] J. Guckenheimer, Sensitive dependence to initial conditions for one-dimensional maps, Comm. Math. Phys. 70 (1979), no. 2, 133-160. MR 82c:58037. Zbl 429.58012.

[7] M. Y. Lyubich, Non-existence of wandering intervals and structure of topological attractors of one-dimensional dynamical systems. I. The case of negative Schwarzian derivative, Ergodic Theory Dynamical Systems 9 (1989), no. 4, 737-749. MR 91e:58100. Zbl 665.58023. 
[8] M. Martens, W. de Melo, and S. van Strien, Julia-Fatou-Sullivan theory for real onedimensional dynamics, Acta Math. 168 (1992), no. 3-4, 273-318. MR 93d:58137. Zbl 761.58007 .

[9] J. Milnor and W. Thurston, On iterated maps of the interval, Dynamical Systems (College Park, MD, 1986-87), Lecture Notes in Math., vol. 1342, Springer, Berlin, New York, 1988, pp. 465-563. MR 90a:58083. Zbl 664.58015.

[10] W. Parry, Symbolic dynamics and transformations of the unit interval, Trans. Amer. Math. Soc. 122 (1966), 368-378. MR 33\#5846. Zbl 146.18604.

Nikos A. Fotiades: SECTION OF MATHEMATICS, DEPARTMENT OF ElECTRICAL AND COMPUTER ENGINEERING, DEMOCRITUS UNIVERSITY OF THRACE, 67100 XANTIH, GREECE

E-mail address: nfotiad@otenet.gr

M. A. Boudourides: Section of MATHEMATiCs, DePARTMENT OF ElECTRICAL AND COMPUTER ENGINEERING, DEMOCRITUS UNIVERSITY OF THRACE, 67100 XANTIH, GREECE

E-mail address: mboudour@duth.gr 


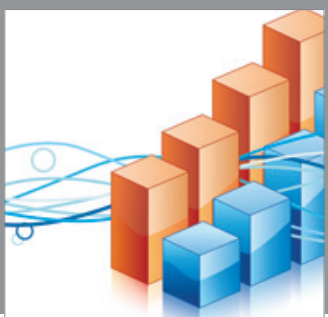

Advances in

Operations Research

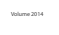

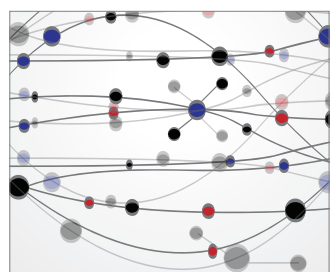

\section{The Scientific} World Journal
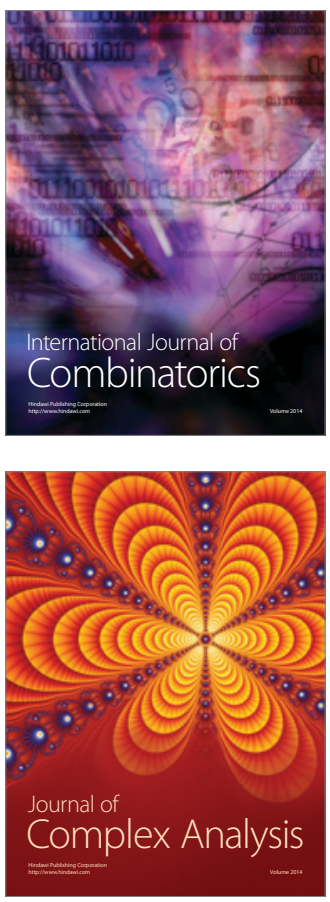

International Journal of

Mathematics and

Mathematical

Sciences
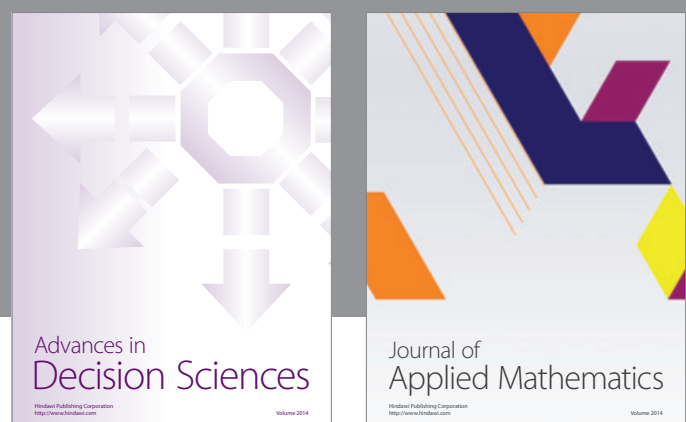

Journal of

Applied Mathematics
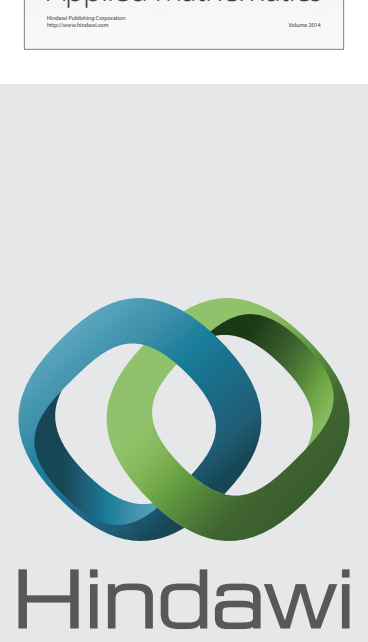

Submit your manuscripts at http://www.hindawi.com
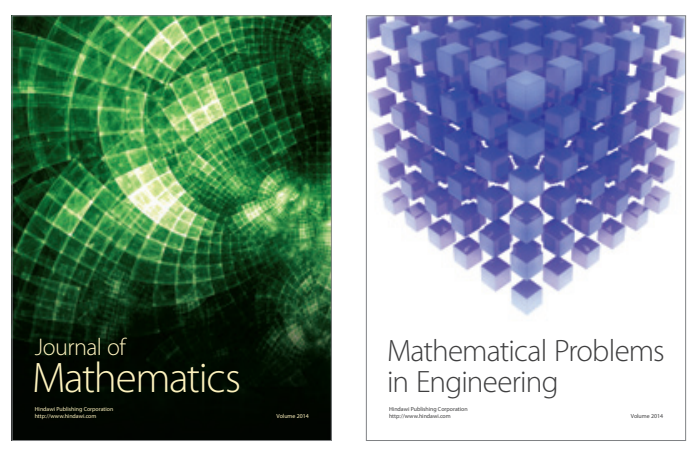

Mathematical Problems in Engineering
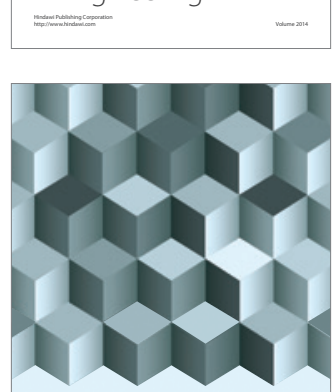

Journal of

Function Spaces
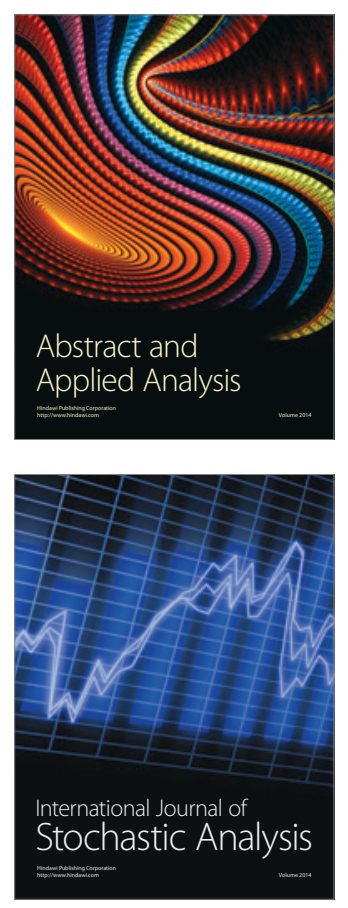

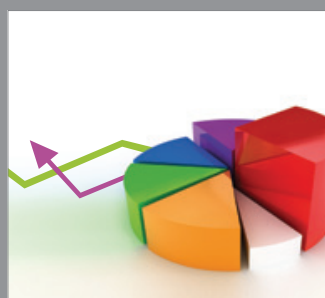

ournal of

Probability and Statistics

Promensencen
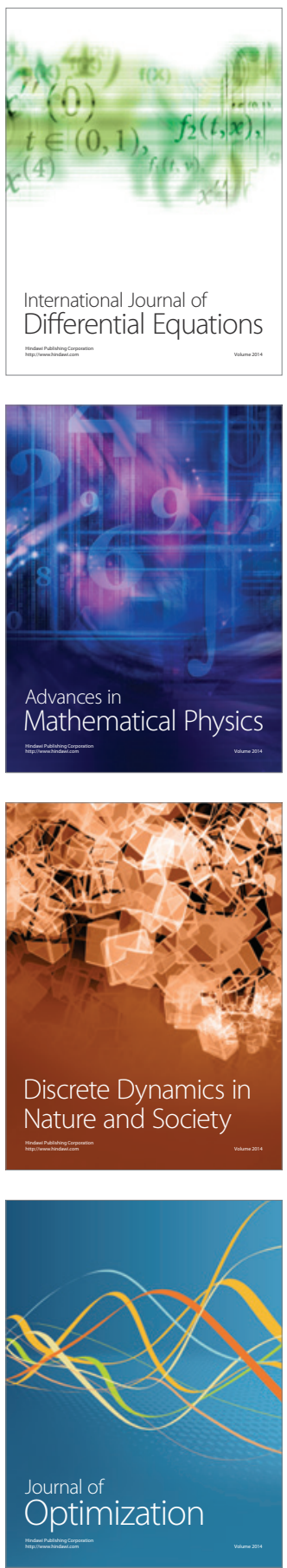